Supporting Information

\title{
In situ measurement of voltage-induced stress in conduct- ing polymers with redox-active dopants
}

Sujat Sen, ${ }^{\mathrm{a}}$ Sung Yeol Kim, ${ }^{\mathrm{b}}$ Lia R.Palmore, ${ }^{\mathrm{b}}$ Shenghua Jin, ${ }^{\mathrm{b}}$ Nitin Jadhav, ${ }^{\mathrm{b}}$ Eric Chason, ${ }^{\mathrm{b}}$ and G. Tayhas R.Palmore ${ }^{\mathrm{a}, \mathrm{b}, *}$

${ }^{\mathrm{a}}$ Department of Chemistry and ${ }^{\mathrm{b}}$ School of Engineering, Brown University, Providence, RI, USA, 02912

*E-mail: Tayhas_Palmore@brown.edu 

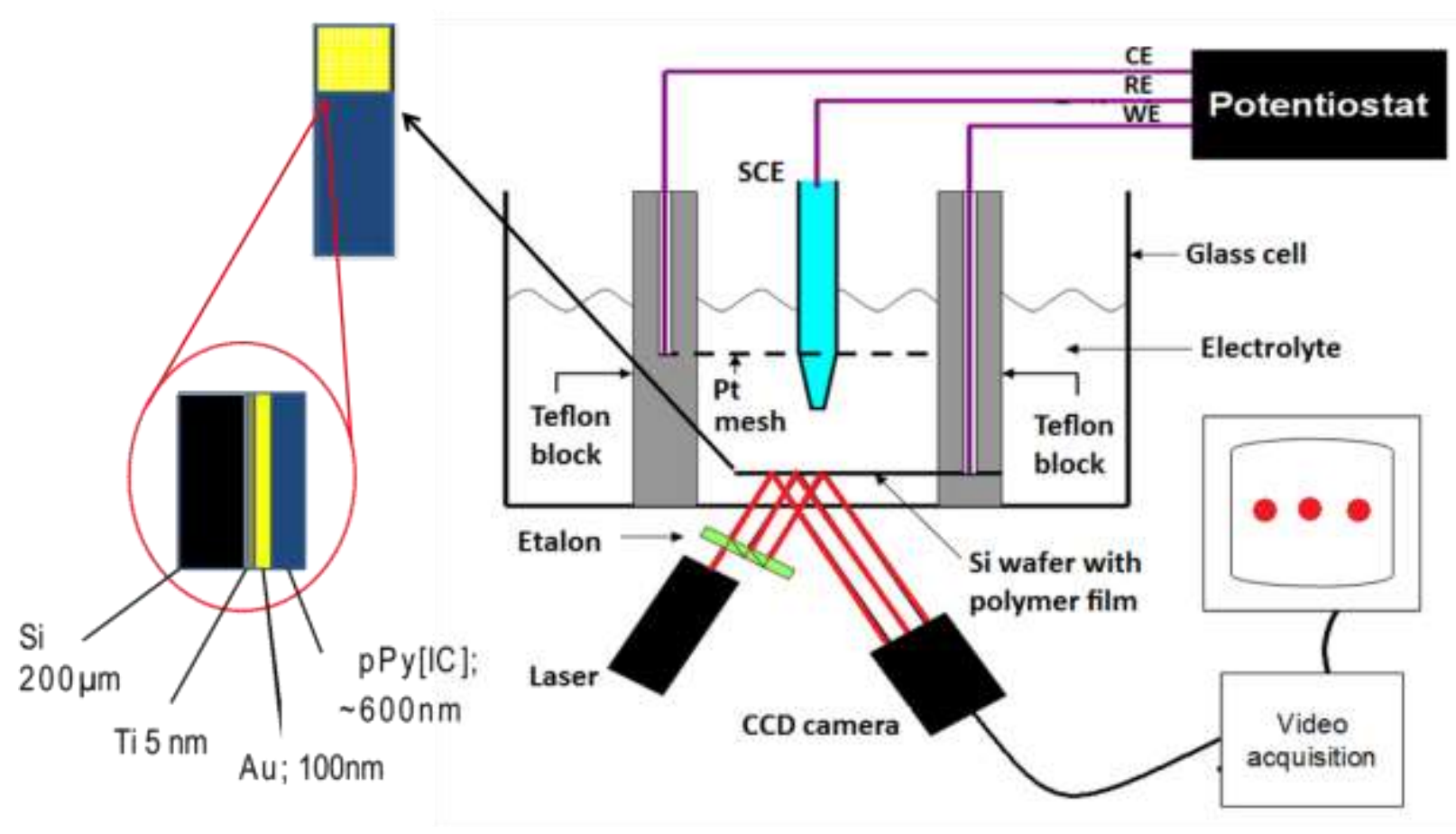

Figure S1. The experimental setup for in-situ measurement of voltage-induced stress in conducting polymer films using MOSS.

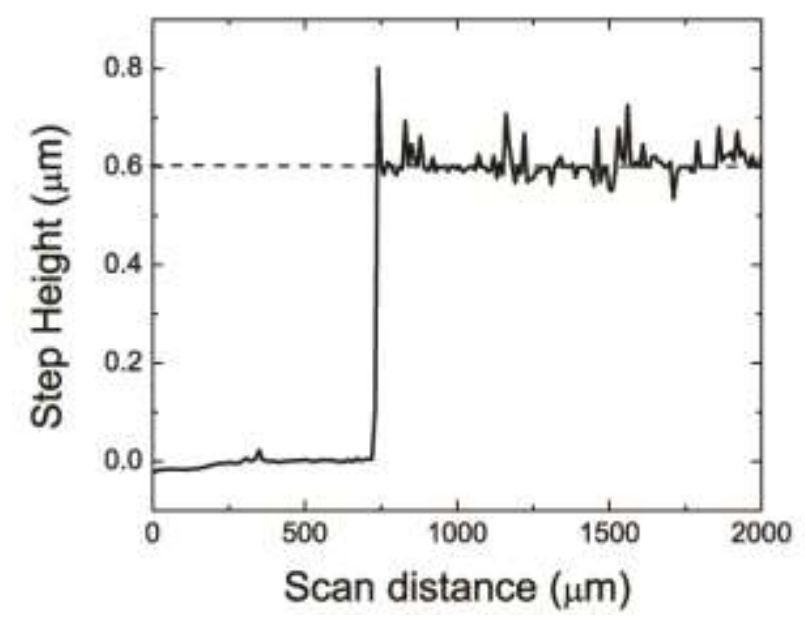

Figure S2. Thickness of a typical pPy[IC] film measured by the Dektak3 surface profilometer. 


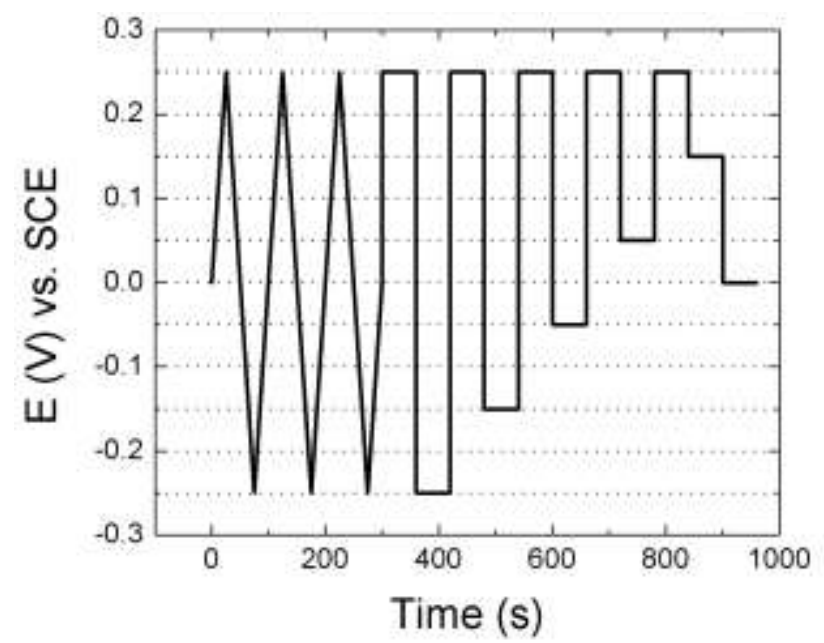

Figure S3. Experimental protocol for electrochemical conditioning.

The inset of Fig. 4a and 6a (hereon referred to as a 'MOSS cycle') show the applied potential protocol that induced the stress evolution in the film. This protocol includes a potential sweep as well as potential pulses between oxidized and reduced states. Specifically, three cycles of a potential sweep (cyclic voltammetry, CV) at $10 \mathrm{mV} / \mathrm{s}$ was applied as follows: 0 to $+0.25 \mathrm{~V}$ to $-0.25 \mathrm{~V}$ to $0 \mathrm{~V}$ vs. SCE. Subsequently, potential steps (chronoamperometry) were applied subsequent to a conditioning potential of $+0.25 \mathrm{~V}$ vs. SCE for $60 \mathrm{~s}: 1)-0.25 \mathrm{~V}$ for $60 \mathrm{~s}$; 2) $-0.15 \mathrm{~V}$ for $60 \mathrm{~s}$; 3) $-0.05 \mathrm{~V}$ for $60 \mathrm{~s} ; 4)+0.05 \mathrm{~V}$ for $60 \mathrm{~s} ; 5$ ) $+0.15 \mathrm{~V}$ for $60 \mathrm{~s}$; 6) $0.0 \mathrm{~V}$ for $60 \mathrm{~s}$ without condition potential. As a result, $1 \mathrm{MOSS}$ cycle corresponds to 3 CVs (300 seconds) +11 constant potential steps (660 seconds) for a total of 960 seconds; 50 cycles corresponds to 48000 seconds. After about $14 \mathrm{hrs,}$ pPy[IC] was found to delaminate from the electrode if the applied potential was more negative than $-0.25 \mathrm{~V}$ vs. SCE. The rate of delamination was dependent on the potential applied. For example, applying a constant potential of (or cycling down to) $-0.8 \mathrm{~V}$ led to instant delamination of the film whereas an applied potential of $-0.5 \mathrm{~V}$ resulted in slower delamination after ca. 25 MOSS cycles. Therefore, a potential window of -0.25 to $+0.25 \mathrm{~V} \mathrm{SCE}$ was chosen to study the film to prevent delamination of films over extended periods of time (over 100 MOSS cycles). 


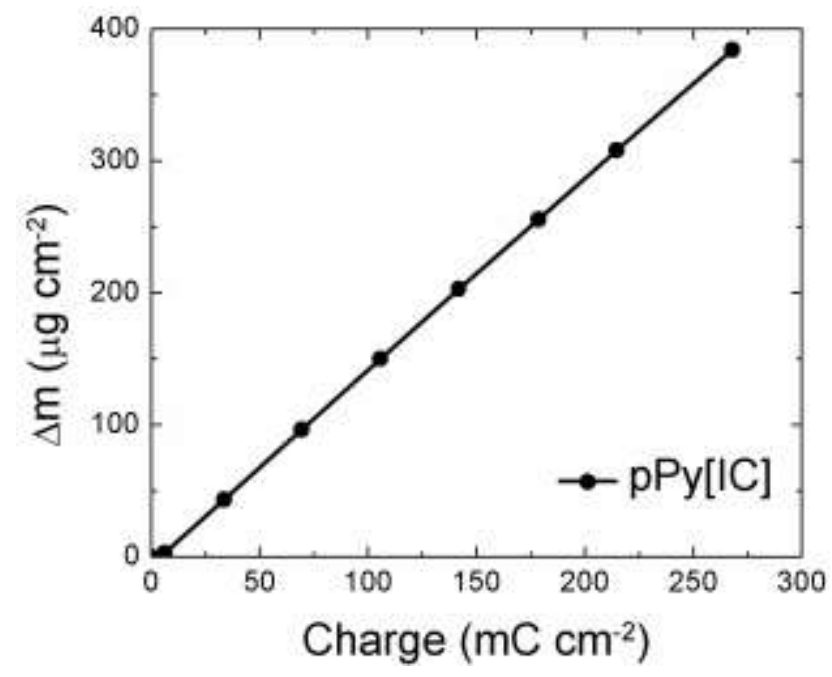

Figure S4. Plot of $\Delta \mathrm{m}$ vs. charge density for the electrosynthesis of a pPy[IC] film on a $\mathrm{Au} / \mathrm{Ti}$-coated $\mathrm{Si}$ substrate.
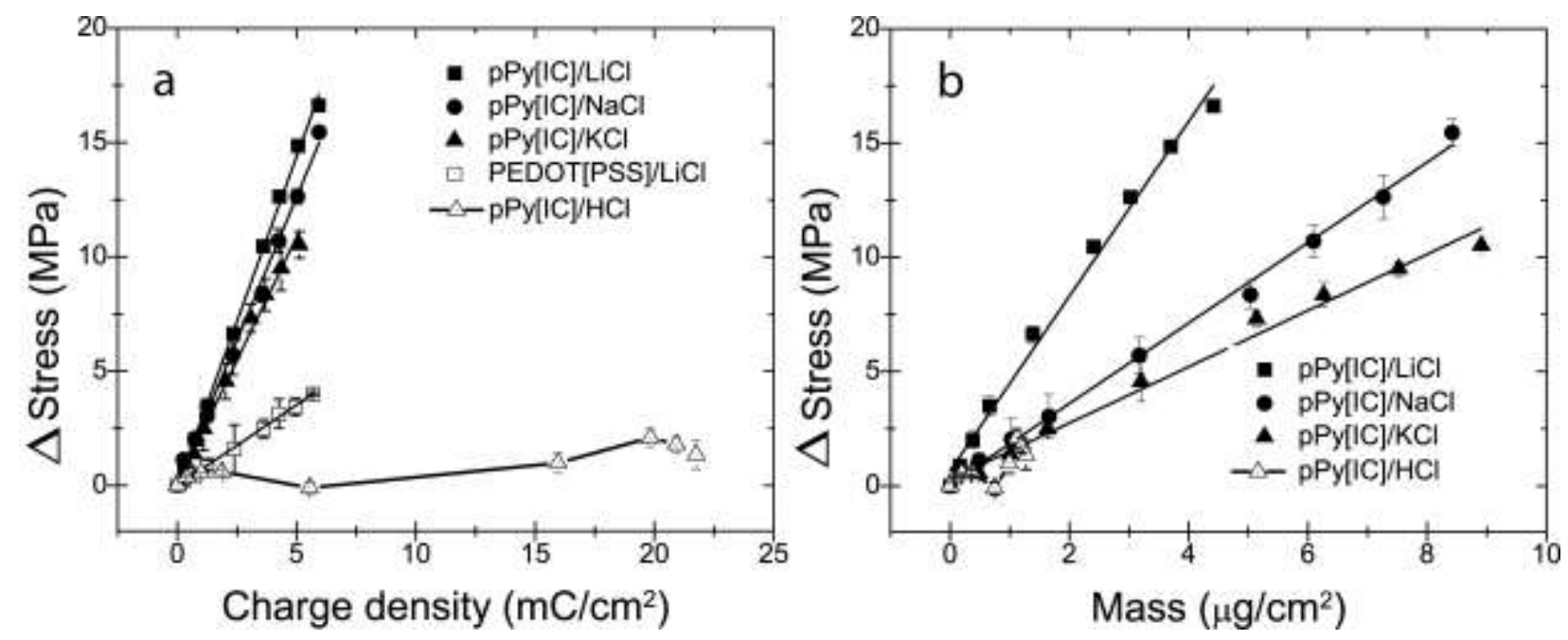

Figure S5. (a) Plot of stress as a function of charge during potential cycling between $0.25 \mathrm{~V}$ to $-0.25 \mathrm{~V}$ during the 50th MOSS cycle in different electrolytes. Data for PEDOT[PSS] is included for comparison. (b) Plot of stress as a function of mass (measured by QCM) during potential cycling between $0.25 \mathrm{~V}$ to $0.25 \mathrm{~V}$ during the 50th MOSS cycle in different electrolytes. 


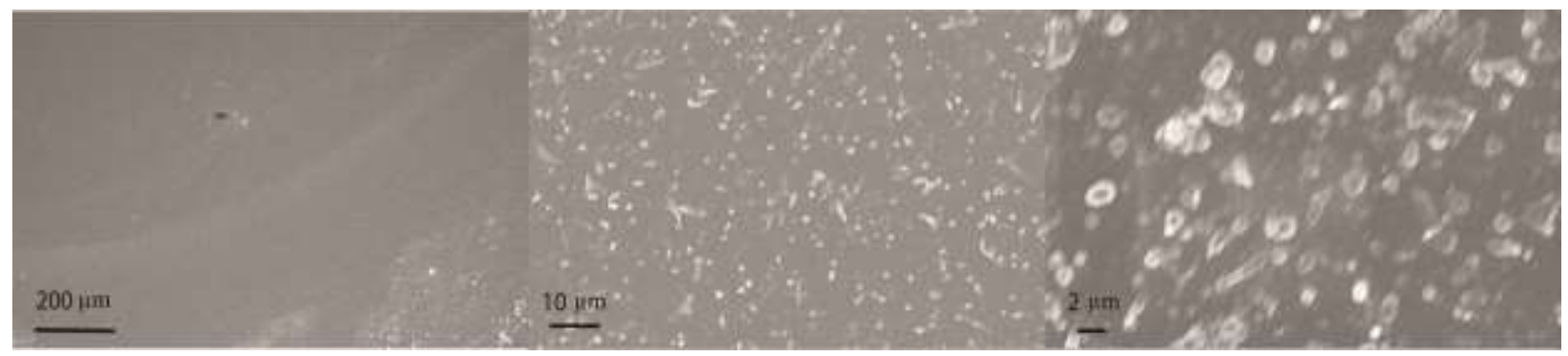

Figure S6. SEM images of an electrodeposited pPy[IC] film.

Table S1. Mass of ions and solvent exchanged in a film of pPy[IC] during the reductive sweep from $+0.25 \mathrm{~V}$ to $-0.25 \mathrm{~V}$ in the 50 th MOSS cycle corresponding to Figure 4.

\begin{tabular}{|c|c|c|c|c|c|c|c|}
\hline ion & $\begin{array}{c}\mathbf{m}_{\text {total }} \\
(\boldsymbol{\mu g})\end{array}$ & $\begin{array}{c}\mathbf{Q}_{\text {red }} \\
(\mathbf{m C})\end{array}$ & $\begin{array}{c}\text { moles of } \\
\mathbf{i o n s} \\
\left(\mathbf{Q}_{\text {red }} / \mathbf{F}\right)\end{array}$ & $\begin{array}{c}\text { molecular } \\
\mathbf{w t . ~ o f ~ i o n ~} \\
(\mathbf{g} / \mathbf{m o l})\end{array}$ & $\begin{array}{c}\text { mass of } \\
\text { bare ions } \\
\left(\mathbf{m}_{\text {ion }} \mathbf{g}\right)\end{array}$ & $\begin{array}{c}\text { mass of water } \\
(\boldsymbol{\mu g}) \\
\left.\mathbf{m}_{\text {total }}-\mathbf{m}_{\text {ion }}\right)\end{array}$ & $\begin{array}{c}\mathbf{m}_{\text {water }} / \\
\mathbf{m}_{\text {ion }}\end{array}$ \\
\hline $\mathbf{L i}^{+}$ & 5.87 & 7.90 & $8.18 \mathrm{E}-08$ & 6.94 & $5.68 \mathrm{E}-07$ & 5.30 & 9.33 \\
\hline $\mathbf{N a}^{+}$ & 10.93 & 7.84 & $8.13 \mathrm{E}-08$ & 22.98 & $1.87 \mathrm{E}-06$ & 9.06 & 4.84 \\
\hline $\mathbf{K}^{+}$ & 11.97 & 6.80 & $7.05 \mathrm{E}-08$ & 39.09 & $2.75 \mathrm{E}-06$ & 9.22 & 3.35 \\
\hline
\end{tabular}

